\title{
Modelling the transition from dunes to the upper-stage plane bed.
}

\author{
O.J.M. van Duin \& S.J.M.H. Hulscher \\ University of Twente, Enschede, The Netherlands
}

\begin{abstract}
: the equilibrium bed load formulation of our dune evolution model has previously been replaced with separate models for the sediment pick-up and deposition processes. Using this approach enabled the modelling of the transition from dunes to the upper-stage plane bed. By increasing the step length (the average distance travelled by sediment particles) manually this lag increases and the dunes become smoother and lower, until they are so smooth that flow separation no longer occurs and finally the dunes wash out.

In the current version of the model the step length changes automatically. This lets the step length increase with increasing flow strength, in line with previous experimental results. This is tested with a synthetic data set where plane bed conditions were reached. With increasing discharge the flow strength increases, which leads to higher step length and which leads to the washing out of dunes.
\end{abstract}

\section{INTRODUCTION}

Hydraulic roughness values play an important role in correctly determining water levels (Casas et al., 2006; Vidal et al., 2007; Morvan et al., 2008), which is critical for flood management purposes. River dunes increase the hydraulic roughness significantly, because their shape causes form drag. In the figure below a developing dune in a flume can be seen.

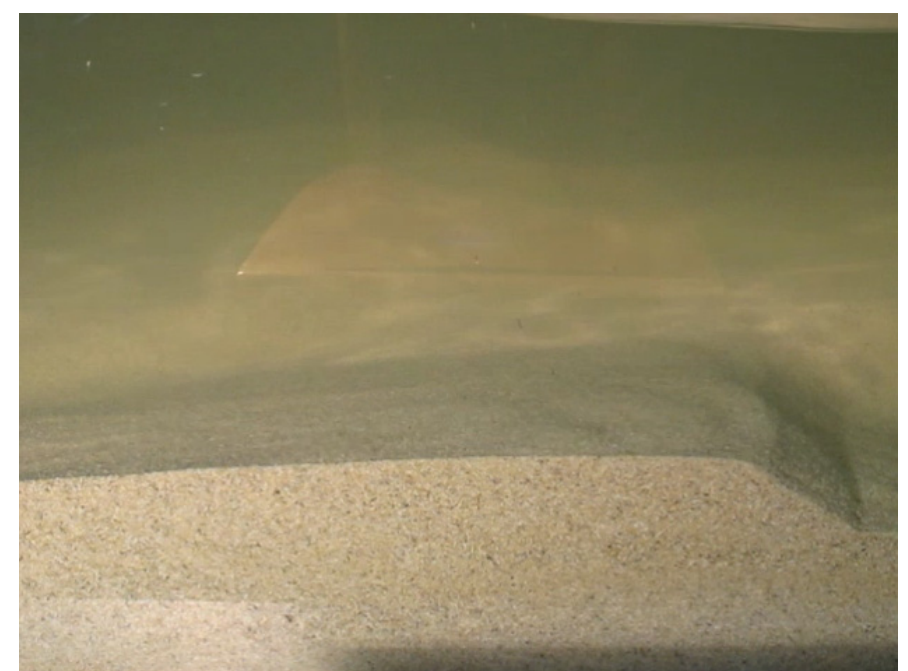

Figure 1: A dune in a flume (flow left to right). The water level is roughly at the top of the figure.

The dune is relatively large compared to the water level, which shows that the dune is a significant bed disturbance and affects the flow. Because of their significant impact on hydraulic roughness, water level forecasts during a high river water discharge depend on accurate predictions of the evolution of river dune dimensions. One aspect of this is the correct prediction of a transition to upper-stage plane bed conditions. Only a few relatively complex dune evolution models are able to model this transition for flume conditions, but no dune evolution model has been able to model such a transition for river conditions. Regardless of the latter, when applied on the large spatial domain of a river segment relatively complex models will lead to too large computation times in the context of flood early-warning systems.

Therefore the aim of this paper is to develop a relatively simple and physics-based dune evolution model that is able to capture proper dune dimensions and dynamic behaviour under transitional conditions. In effect, we want to be able to model dune behaviour under varying discharge from the lower stage plane bed to the upper stage plane bed. This model should work under flume and field conditions, and should predict transitions at the appropriate moments (at critical values for grain shear stress, suspension number, etc.).

In the past, many approaches have been used to model dune dimensions, varying from equilibrium dune height predictors (e.g. Yalin, 1964; Allen, 1978; Van Rijn, 1984) to different forms of stability analyses (e.g. Kennedy, 1963; Engelund, 1970; Fredsøe, 1974; Yamaguchi \& Izumi, 2002). Recently, models have been developed that calculate the turbulent flow field over bedforms, in some cases in combination with morphological computations (e.g. Nelson et al., 2005; Tjerry \& Fredsøe, 2005; Shimizu et al., 2009; Nabi et al., 2010, 2013). These mod- 
els are valuable to study detailed hydrodynamic processes, but are computationally intensive which makes them unsuitable for applications on the river scale.

To be able to efficiently predict dune dimensions over the time-scale of a flood wave Paarlberg et al. (2009) developed a model in which the flow and sediment transport at the flow separation zone is parameterized instead of using full hydrodynamic equations. This model is able to predict the evolution of dunes from small initial disturbances up to equilibrium dimensions with limited computational time and good accuracy. In addition, this model has been coupled with an existing hydraulic model to form a 'dynamic roughness model' (Paarlberg et al., 2010). Results are promising, as the coupled model clearly shows the expected hysteresis effects in dune roughness and water levels and different behaviour of sharp-peaked versus broad-peaked flood waves within the dune regime (Paarlberg et al., 2010).

As Nakagawa \& Tsujimoto (1980) argue, a lag distance between flow properties (and thereby bed shear stress) and sediment transport is the principal cause of bed instability and thereby regime transitions. One of the factors contributing to this is the probability distribution of sediment particle step length, which is the distance travelled from dislodgement to rest according to Einstein (1950). This creates a phase-lag effect which is not taken into account in the transport formula like that of MeyerPeter and Müller (1948) of the original model of Paarlberg et al. (2009), and which made it impossible to model a transition to upper-stage plane bed. The pick-up and deposition model Nakagawa \& Tsujimoto (1980) propose to determine bed load transport, inherently allows a phase-lag effect over distance. The deposition of sediment away from the pick-up point is determined by using a distribution function that relies on the mean step length. This pick-up and deposition model had already been used in the dune evolution model of Shimizu et al. (2009), with good results (regarding prediction of dunes and upper-stage plane bed).

Therefore, an extension of the Paarlberg et al. (2009) model has been made to make it possible able to predict a transition to upper-stage plane bed. In Van Duin et al. (2014, submitted) it is shown that by replacing the transport formula of Meyer-Peter and Müller (1948) with the pick-up and deposition model of Nakagawa \& Tsujimoto (1980) we get good results in the dune regime. Furthermore, Van Duin et al. (2014, submitted) are able to wash out dunes with this model as well, signifying the potential for prediction upper-stage plane bed. However, the selection of a physics-based step length is still an issue.

High values of the step length will lead to the washing out of dunes within the model of Paarlberg et al. (2009), with the newly implemented bed load model as presented in Van Duin et al. (2014, submit- ted)/ The question arises whether the step length should be varied over just time (because of changing flow regime) or along the dune as well (because of local variation in shear stress). From preliminary experimental results we have found that mean step lengths in the trough of a dune are very similar to mean step lengths at the crest of a dune (Van Duin et al., 2012), which suggests that variations along the dune are limited. This is probably because though the turbulence-averaged bed-shear stress in the trough is lower, the extreme turbulent events (e.g. due to flow reattachment) are much stronger. The mean step lengths therefore become more or less the same along the dune, which implies that only a variation of step length over time is needed.

The main research question of this paper is how to calculate the step length from the hydrodynamic data, so that we can replicate the validation case of Shimizu et al. (2009). This validation case is similar to experiments done in flumes.

The organisation of the paper is as follows. In chapter 2 the set-up of the model and the different sub models will be discussed. Chapter 3 shows the calculation case, and chapter 4 shows results of the model runs. In chapters 5 and 6 the discussion and conclusion are presented.

\section{DUNE MODEL}

\subsection{General set-up}

The basis of the present model is the dune evolution model developed by Paarlberg et al. (2009). Paarlberg et al. (2009) extended the process-based morphodynamic sand wave model of Németh et al. (2006), which is based on the numerical model of Hulscher (1996), with a parameterization of flow separation, to enable simulation of finite amplitude river dune evolution.

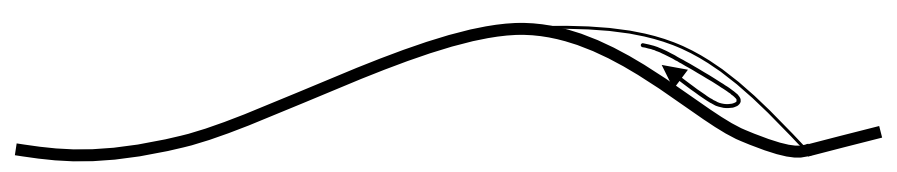

Figure 2: Schematization of a dune (flow left to right)

Flow separation is forced in the model when the leeside slope exceeds $10^{\circ}$. To model flow separation (see Figure 2) behind the dune and its effect on flow, the bed shear stress distribution and the sediment transport is included in a parameterized way using experimental data of turbulent flow over twodimensional subaqueous bedforms (Paarlberg et al. 2007). In the flow separation zone the bed shear stress is assumed to be zero and all the sand transport that reaches the crest of the dune is avalanched under the angle of repose on the leeside of the dune (Paarlberg et al., 2009). This enables the model to predict river dunes with their characteristic 
shape and realistic dimensions without resolving the complex recirculating flow in the flow separation zone.

The model consists of a flow module, a sediment transport module and a bed evolution module which operate in a decoupled way. The model simulates a single dune which is assumed to be in an infinite train of identical dunes. Therefore periodic boundary conditions are used. The dune length is determined by the simple relation of Van Rijn (1984). The dune length is 7.3 times the water depth, which follows from the relations for dune height and length of Van Rijn (1984) as presented by Julien \& Klaassen (1995).

\subsection{Flow model}

In general the flow is forced by the difference in water level across the domain. Though the water depth at the start and end of domain are the same due to the periodic boundary conditions, the water level differs because the domain is sloped. The average bed level is taken as zero and has a slope (this average bed slope is an input parameter for the model). By solving the flow equations with a certain average water depth a discharge is found. The average water depth is adjusted until this discharge matches the discharge given as input.

\subsection{Governing equations}

The flow in the model of Paarlberg et al. (2009) is described by the two-dimensional shallow water equations in a vertical plane (2-DV), assuming hydrostatic pressure conditions. For small Froude numbers the momentum equation in vertical direction reduces to the hydrostatic pressure condition, and that the time variations in the horizontal momentum equation can be dropped. The governing model equations that result are shown in equations (1) and (2).

$u \frac{\partial u}{\partial x}+w \frac{\partial u}{\partial z}=-g \frac{\partial \zeta}{\partial x}+A_{v} \frac{\partial^{2} u}{\partial z^{2}}+g i$

$\frac{\partial u}{\partial x}+\frac{\partial w}{\partial z}=0$

The velocities in the $\mathrm{x}$ and $\mathrm{z}$ directions are $u$ and $w$, respectively. The water surface elevation is denoted by $\zeta, i$ is the average channel slope, $g$ is the acceleration due to gravity, and $A_{v}$ denotes the constant vertical eddy viscosity. The computational domain is shown in Figure 3.

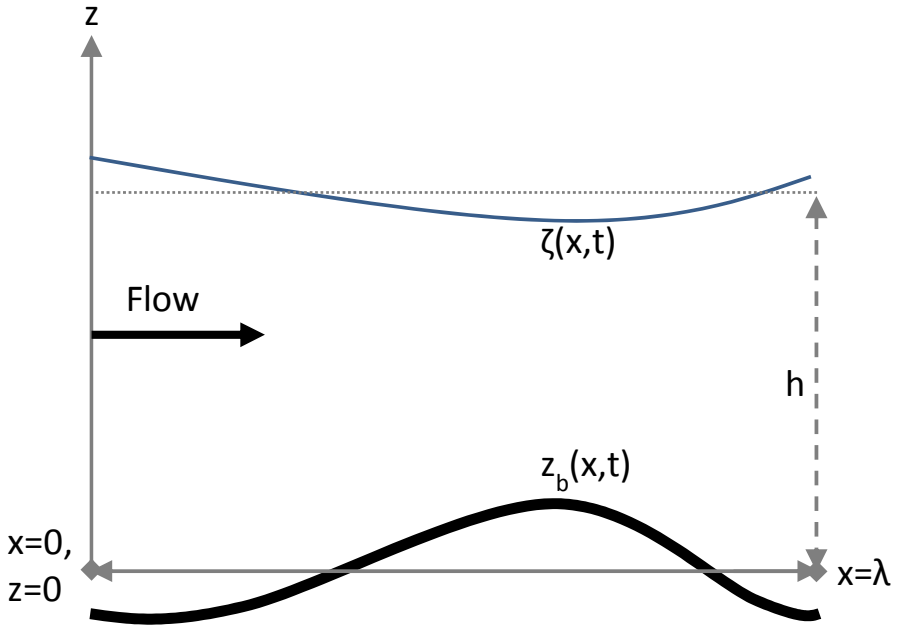

Figure 3: The computational domain

In Figure $3 \lambda$ denotes the dune length (in $\mathrm{m}$ ), $h$ is the domain-averaged water depth (in $\mathrm{m}$ ) and $z_{b}$ is the bed level relative to the $\mathrm{x}$-axis (in $\mathrm{m}$ ). The flow is forced in the domain because the $\mathrm{x}$-axis is actually at a slope $i$ with regard to the real horizontal plane, creating a water level difference along the domain.

\subsubsection{Boundary conditions}

The boundary conditions are defined at the water surface $(\mathrm{z}=h+\zeta)$ and at the bed $\left(\mathrm{z}=z_{b}\right)$. The boundary conditions at the water surface, equation (3) represents no flow through the surface and equation (4) means no shear stress at the surface. The kinematic boundary condition at the bed, equation (5) yields that there is no flow through the bed.

$$
\left.\frac{\partial u}{\partial z}\right|_{z=h+\zeta}=0
$$

$\left.u \frac{\partial \zeta}{\partial x}\right|_{z=h+\zeta}=w$

$\left.u \frac{\partial z_{b}}{\partial x}\right|_{z=z_{b}}=w$

In order to close the turbulence model, we assume a time- and depth-independent eddy viscosity. In order to represent the bed shear stress correctly for a constant eddy viscosity, a partial slip condition at the bed, equation (6) is necessary.

$\tau_{b}=\left.A_{v} \frac{\partial u}{\partial z}\right|_{z=z_{b}}=S u_{b}$

In equation (6) $\tau_{b}\left(\mathrm{~m}^{2} / \mathrm{s}^{2}\right)$ represents the volumetric bed shear stress, $u_{b}(\mathrm{~m} / \mathrm{s})$ is the flow velocity along the bed, and the resistance parameter $S(\mathrm{~m} / \mathrm{s})$ controls the resistance at the bed. For more details about the model equations and numerical solution procedure, reference is made to Paarlberg et al. (2009), Van den Berg et al. (2012). 


\subsection{Bed load sediment transport model}

The pick-up and deposition model of Nakagawa \& Tsujimoto (1980) uses the following formulae to determine bed load transport. Pick-up of sediment (probability of a particle being picked up in $\mathrm{s}^{-1}$ ) is determined by

$p_{s}(x)=F_{0} \sqrt{\frac{\Delta g}{D_{50}}} \theta(x)\left(1-\frac{\theta_{c}}{\theta(x)}\right)^{3}$

where $F_{0}=0.03, \theta$ is the Shields parameter, $\theta_{c}$ is the critical Shields parameter.

The local, critical volumetric bed shear stress $\tau_{c}(x)$, corrected for bed slope effects, is given by the following equation.

$$
\tau_{c}(x)=\tau_{c 0} \frac{1+\eta \frac{\partial z_{b}}{\partial x}}{\sqrt{1+\left(\frac{\partial z_{b}}{\partial x}\right)^{2}}}
$$

with $\tau_{c 0}$ the critical volumetric bed shear stress for flat bed, defined by equation (9). In this equation $\theta_{c 0}$ is the critical Shields parameter for flat bed and $D_{50}$ is the median grain size.

$\tau_{c 0}=\theta_{c 0} g \Delta D_{50}$

Deposition at a location is determined by adding all the sediment that arrives at that specific location. So, in order to determine the deposition at a certain location $\mathrm{x}$ the distribution of picked up sediment from all upstream locations is needed. The determination of deposition is done by applying the following formula:

$$
p_{d}(x)=\int_{0}^{\infty} p_{s}(x-s) f(s) d s
$$

where the distribution $f(\mathrm{~s})$ determines the fraction of sediment that is deposited a distance s away from the pick-up point $(\mathrm{x}-\mathrm{s})$. The distribution function is defined as follows:

$$
f(s)=\frac{1}{\Lambda} \exp \left(\frac{-s}{\Lambda}\right)
$$

By using this function, all the sediment that has been picked up at certain location is deposited between that location and 5 times the step length in downstream direction. Finally the transport gradient is determined as follows:

$\frac{d q_{b}(x)}{d x}=D_{50}\left[p_{s}(x)-p_{d}(x)\right]$

To summarize, the entire calculation process is as follows. First the bed shear stress is determined from flow characteristics. Then the pick-up of sediment along the dunes follows from bed shear stress. With an exponential decay function the deposition of sediment away from each pick-up point is determined.
The difference between sediment deposition and pick-up determines the net transport gradient along the dune.

\subsection{Step length}

\subsubsection{Definition of step length}

To calculate how the sediment is distributed away from each pick-up point we need to calculate the mean step length the sediment particles take. Step length is defined by Einstein (1950) as

$\Lambda=\alpha D_{50}$

where $\alpha$ is a non-dimensional step length parameter. Francis (1973), Fernandez Luque \& Van Beek (1976) and Sekine \& Kikkawa (1984) have done experiments to determine the dependency of among others bed load transport, particle velocity and step length on various parameters with moving sand along a plane bed. This data shows a range of approximately 40 to 240 times the particle diameter, for values of $u^{*} / w_{s}$ from about 0.18 to 0.35 . From this data different step length models are derived by various authors. Here we will discuss two methods, and present a third method (based on the first two) that is implemented in our dune evolution model.

\subsubsection{Sekine \& Kikkawa (1992) step length model}

Sekine \& Kikkawa (1992) have used the aforementioned data sets to verify a numerical model of saltation of particles. They found that all computed step length values are no more than two times larger or smaller than the observed values. Their predictions for the thickness of the saltating bed load layer closely match the data of Sekine \& Kikkawa (1984); the particles remain within a few grain diameters from the bed. They further show that in their calculations the mean step length varies between about 10 and about 350 times the particle diameter, directly proportional to friction velocity $u^{*}\left(u^{*}=(\tau / \rho)^{1 / 2}\right)$ and inversely with settling velocity $w_{s}$. The suspension parameter $u^{*} / w_{s}$ ranges from about 0.15 to 0.28 in this set of calculations. The relation between these parameters and the non-dimensional step length $\alpha$ is

$$
\alpha=\frac{\Lambda}{D_{50}}=\alpha_{2}\left(\frac{u_{*}}{w_{s}}\right)^{3 / 2}\left(1-\frac{u_{*_{c}} / w_{s}}{u_{*} / w_{s}}\right)
$$

where $\alpha_{2}=3.0 * 10^{3}$ and $u^{*}{ }_{c}$ is the critical friction velocity $\left(u_{c}^{*}=\tau_{c}{ }^{1 / 2}\right.$; note that we again use volumetric bed shear stress).

\subsubsection{Shimizu et al. (2009) step length model}

Shimizu et al. (2009) use the experimentally found minimum and maximum value of non-dimensional 
step length $\alpha$ to derive a relation between $\alpha$ and dimensionless grain shear stress $\theta^{\prime}$. For values of $\theta^{\prime}$ between zero and 0.5 (the dune regime), $\alpha$ is constant at the minimum value. For values of $\theta^{\prime}$ above 0.8 (the upper stage plane bed regime), $\alpha$ is constant at the maximum value. In the transitional regime $(\theta$, from 0.5 to 0.8$), \alpha$ is linearly interpolated. There is no dependency on sediment parameters.

\subsubsection{Choice of step length model}

The step length model strikes a balance between the conceptual model of Shimizu et al. (2009) and the more physics-based model of Sekine \& Kikkawa (1992). Using the former led to such low values for the step length that no upper stage plane bed occurred, while using the latter led to such high values that no dunes were able to grow. To solve this unwanted behaviour we explored different adjustments to the parameters of the Shimizu et al. (2009) step length model, where the Sekine \& Kikkawa (1992) model served as an upper limit for the chosen values.

We follow Shimizu et al. (2009) between $\theta^{\prime}=0$ and $\theta^{\prime}=0.5$. Next we set the value of $\alpha$ to 700 for $\theta^{\prime}=0.8$. Between $\theta^{\prime}=0.5$ and $\theta^{\prime}=0.8$ we linearly interpolate the values of $\alpha$. Subsequently we let the step length increase for $\theta^{\prime}$ 'values above 0.8 (with the same slope as between $\theta^{\prime}=0.5$ and $\theta^{\prime}=0.8$ ). This leads to a step length model that works well within our dune evolution model. In the figure below the currently used compared step length model is compared with the Shimizu et al. (2009) and the Sekine \& Kikkawa (1992) models.

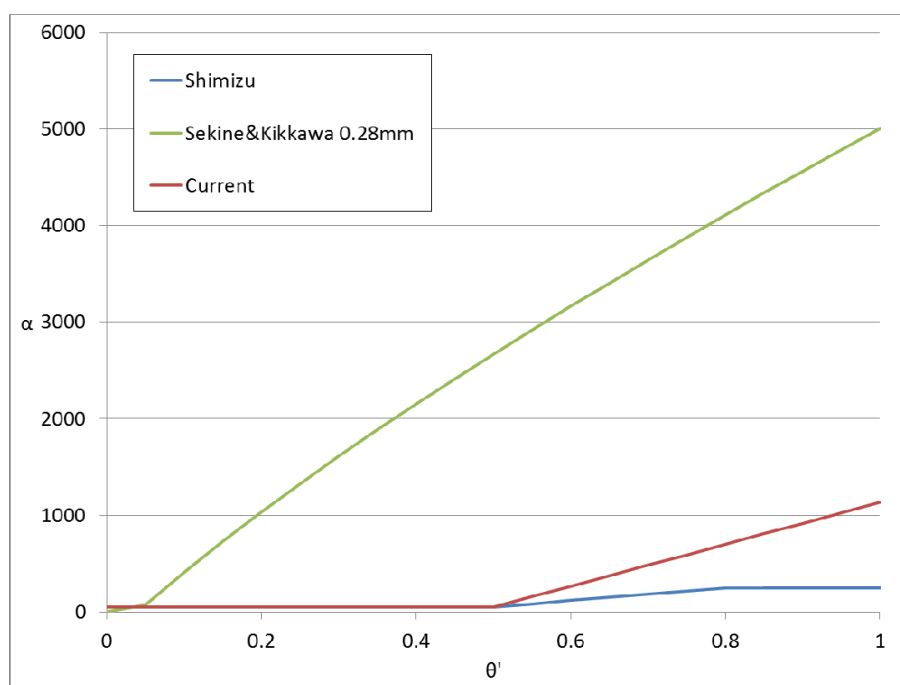

Figure 4: The non-dimensional step length results for varying grain shear stress of the Shimizu et al. (2009), Sekine \& Kikkawa (1992) and the currently used step length models.

It can be seen that our step length model leads to significantly higher non-dimensional step lengths than the Shimizu et al. (2009) step length model, though it's still far below the results obtained with the Sekine \& Kikkawa (1992) model. It should be noted that because the latter model needs a grain di- ameter as input, the results only hold for the median grain diameter from the calculation case presented in the next paragraph. Lines for other grain diameters will therefore change for the Sekine \& Kikkawa (1992) model but not for the other two. The dimensional step length (calculated with equation 13) of course does change with varying grain diameter for all three models.

\subsection{Bed evolution}

The bed evolution is modelled using the Exner equation given by equation (15), where the sediment transport gradient is calculated with equation 12 and $\varepsilon_{p}=0.4$ is the bed porosity.

$\left(1-\varepsilon_{p}\right) \frac{\partial z_{b}}{\partial t}=-\frac{\partial q_{b}}{\partial x}$

After bed evolution the model checks the angle of the bed between every pair of neighbouring calculation points. If necessary, the model avalanches the 'excess' sand so that the angle of the bed is below the angle of repose $\left(30^{\circ}\right)$ everywhere.

\section{CALCULATION CASE}

For validation we use computational scenario A4 as presented by Shimizu et al. (2009). For the model runs they used a $D_{50}$ of $0.28 \mathrm{~mm}$, a slope $i$ of $2 * 10^{-3}$, and a hydrograph as presented below.

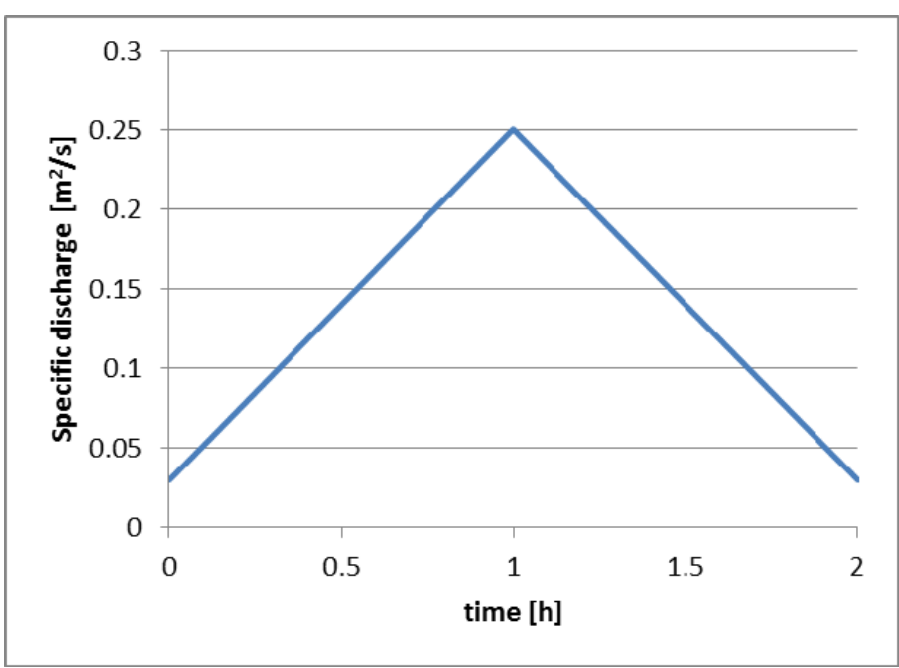

Figure 5: Hydrograph of scenario A4 from Shimizu et al. (2009).

With this scenario Shimizu et al. (2009) show that their model is able to predict transitions at the correct values of grain shear stress. Also the model clearly shows hysteresis effect; the relation between discharge and water depths is significantly different for the rising limb of the hydrograph as it is for the falling limb. The parameters of the scenario are equivalent to a flume scenario. 


\section{RESULTS}

Using scenario A4 from Shimizu et al. (2009) we find the following development of the dune field over time.

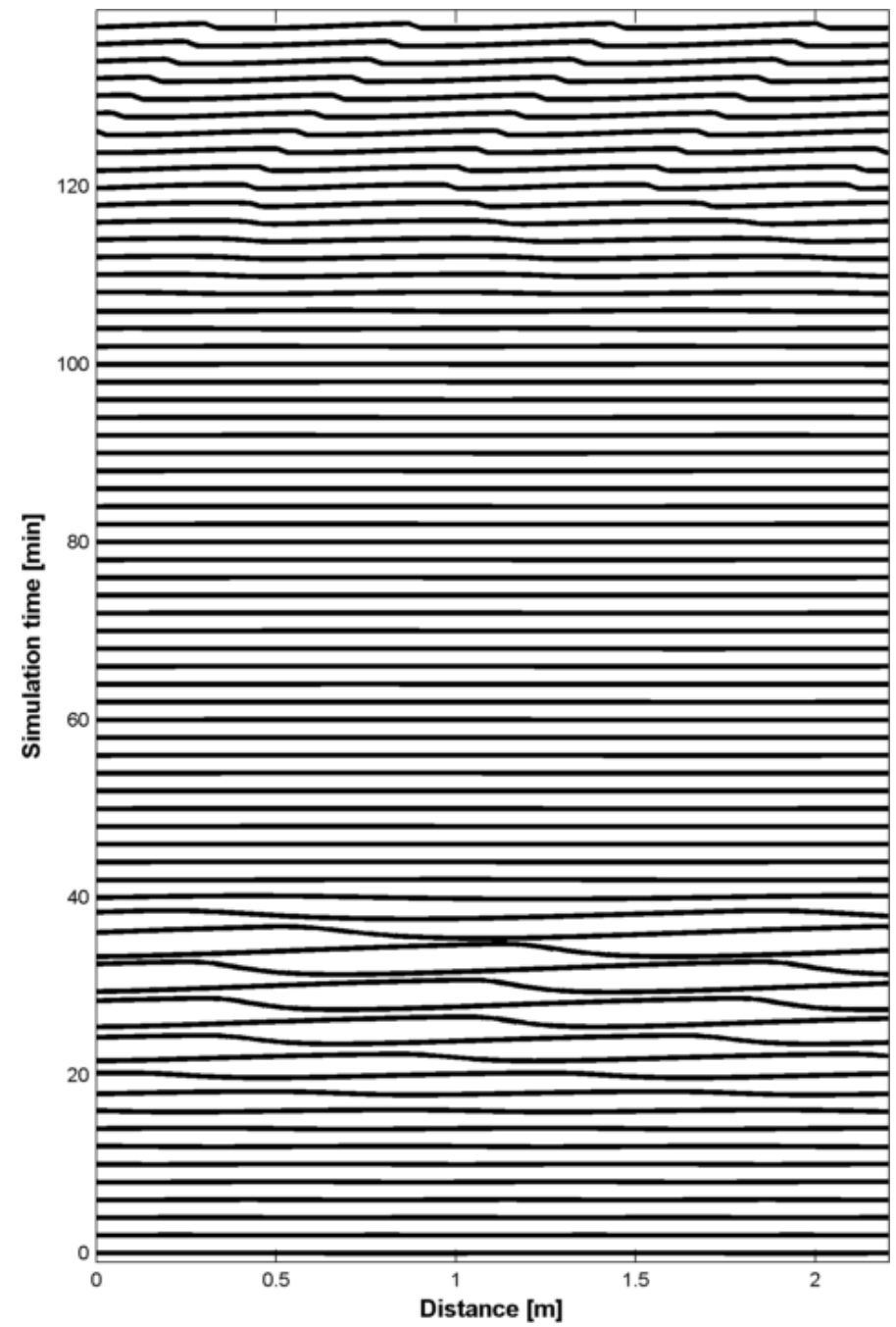

Figure 6: Dune field over time.

Here the washing out and regrowth of the dunes can be clearly observed. It should be noted that the single dune we actually model (see paragraph 2 ) is repeated a few times per moment in time for visualization purposes.

From the same results the development of the dune trough and crest positions (in the vertical) and water depth over time are shown below. As can be seen in the beginning of the run dunes start developing along with increasing discharge. At a certain high discharge the shear stress becomes high enough to select a high step length and the dunes are washed out. Due to the decrease in form drag and thereby total shear stress the water level goes down, despite still rising discharge. The bed remains washed out until the discharge becomes low enough to again select a low step length and dunes start developing again.

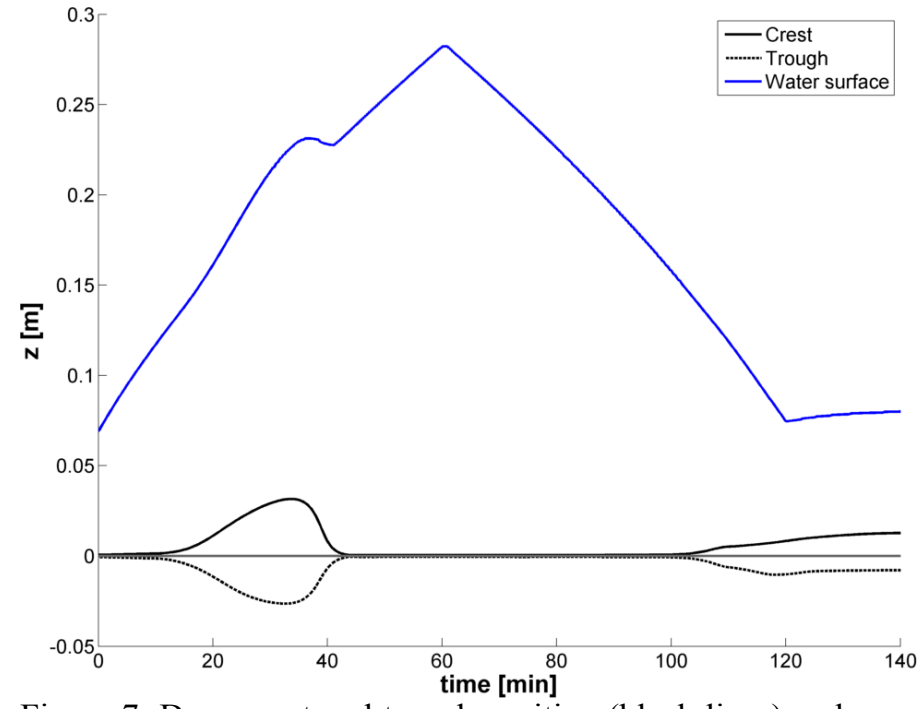

Figure 7: Dune crest and trough position (black lines) and water depth (blue line) over time.

Shimizu et al. (2009) found roughly the same moments of washing out (40 minutes) and new development (100 minutes). The relation between $\alpha$ and the specific discharge for the rising and falling stages of the hydrograph can be seen below.

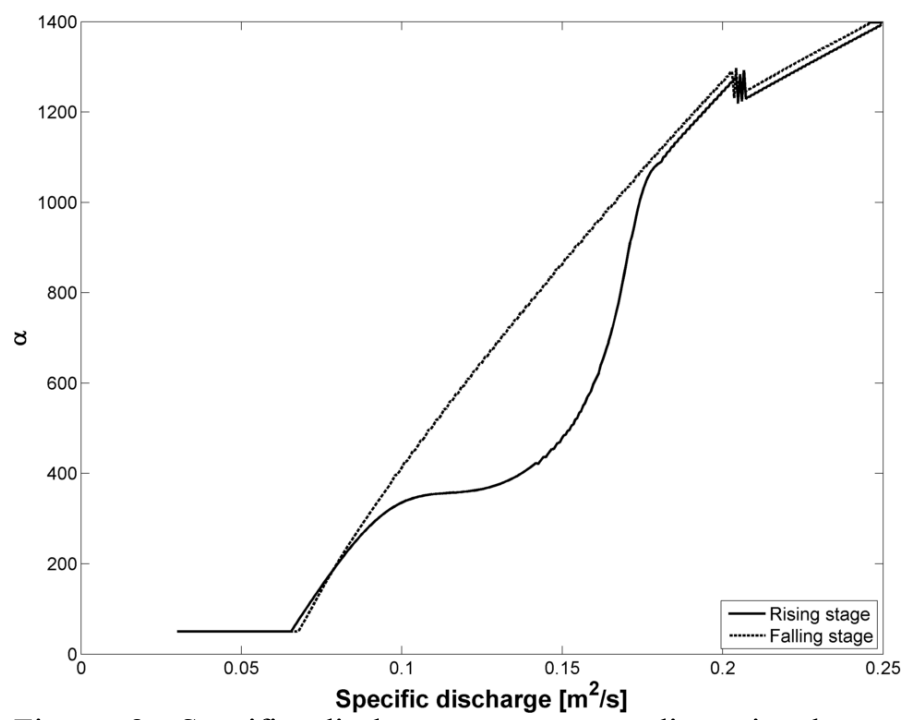

Figure 8: Specific discharge versus non-dimensional step length $\alpha$ separated for the rising and falling stages of the hydrograph.

Here we already observe a hysteresis effect caused by the transition to upper stage plane bed and back to the dune regime. With the same discharge dunes can be present in the rising stage and not in the falling stage. Because of the presence of dunes, a part of the flow power is lost due to form drag. This means the shear stress at the bottom is relatively lower, and a lower step length is selected then when there are no dunes present. The effect of hysteresis on water depth can be seen in the following figure. 


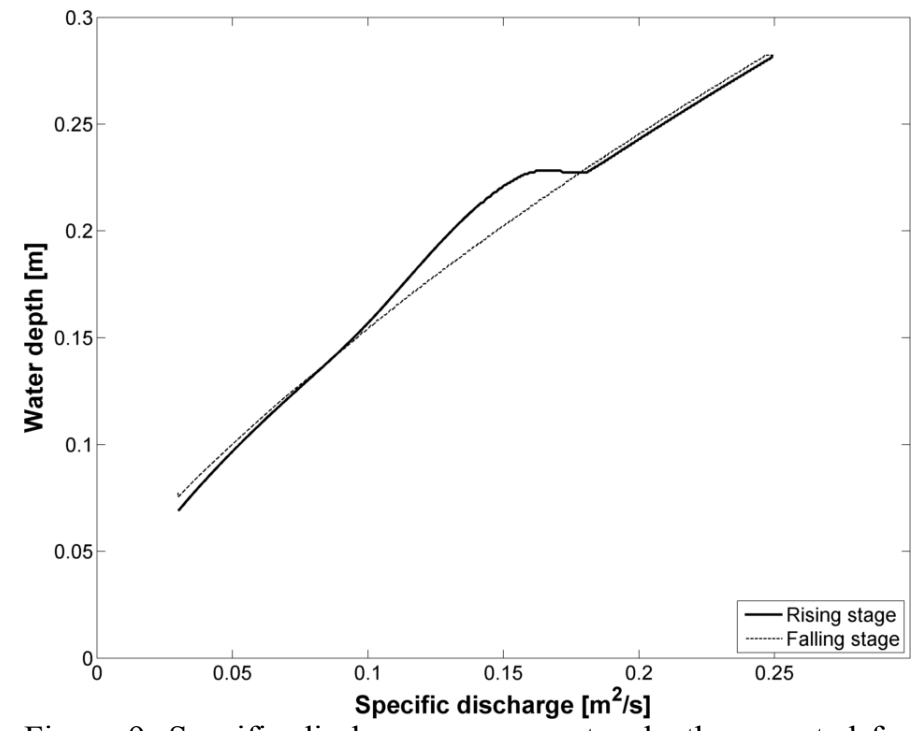

Figure 9: Specific discharge versus water depth separated for the rising and falling stages of the hydrograph.

In the rising part of the hydrograph dunes are able to develop firstly at lower discharges, while in the falling part the dunes start developing at higher discharges. This has a clear effect on the resulting water depths at the same discharge. For example, at a specific discharge of $0.15 \mathrm{~m}^{2} / \mathrm{s}$ the water depth in the rising part is clearly higher than in the falling part. In the rising limb the dunes have had a longer time to grow than in the falling limb, and are therefore higher. Because the dunes are higher, the water depth is higher despite the discharge being the same. Shimizu et al. (2009) clearly see this hysteresis effect as well, though for their model it's more pronounced. They also report generally lower water depths than we find, in the order of $25 \%$ difference. The hysteresis effect can also be seen by looking at the behaviour of the Froude number in the figure below.

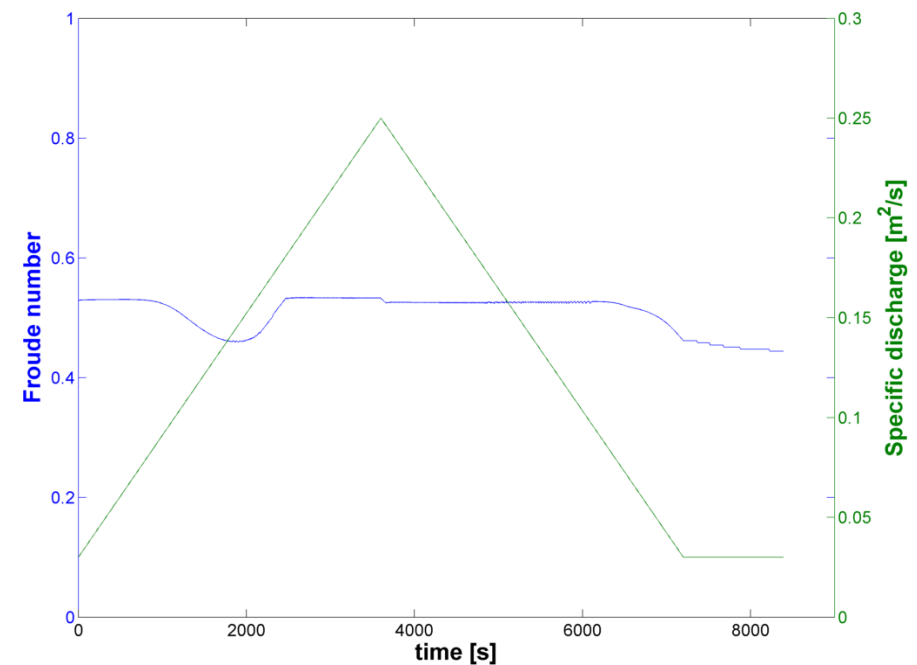

Figure 10: Froude number (left vertical axis) and specific discharge (right vertical axis) versus time.

For the discharges where there is a difference in water depth between the rising and falling stages the Froude number is of course different as well. For example, this can clearly be seen around a specific discharge of $0.15 \mathrm{~m}^{2} / \mathrm{s}$. In the rising stage the Froude number is lower because of the relatively high water depth when compared to the falling stage. Because of differences in water depth, also for the Froude number the differences are more pronounced with the Shimizu et al. (2009) model.

\section{DISCUSSION}

We observed some differences in outcome between our model and the model of Shimizu et al. (2009), i.e. our step lengths have to become significantly larger to reach the transition to an upper-stage plane bed and there are differences of $25 \%$ in water levels. Of course, the models differ in some significant ways. Firstly Shimizu et al. (2009) use a nonhydrostatic flow model with a non-linear k- $\varepsilon$ model for turbulence closure whereas we use a hydrostatic flow model with a constant eddy viscosity as turbulence closure. Secondly their transport module also employs a suspended load pick-up model while we solely focus on bed load. Thirdly, as explained in detail, we use a modified version of their step length model. Pinpointing the exact reason of the differences is hard because the case under review is a 'synthetic' case; we have no actually measured values as a reference to determine which model is closer to the truth. The Shimizu et al. (2009) dune model is more physically complex, which suggest that its results should be better. However, it's still promising that with our relatively simple model we can get quite close to the behaviour predicted by them. This gives us confidence to apply this model to measured scenarios.

It should be noted that suspended transport can play a role in the transition to upper-stage plane bed as well, as it also causes a lag between shear stress and the dune shape. We use higher values for step length than Shimizu et al. (2009) do, and that may be because we do not have a separate suspended transport model. Using only bed load with low step lengths does not lead to strong enough lag, so to find similar behaviour we need to increase the step length and thereby we may be implicitly taking into account the effects of suspended transport as well. It could be interesting to use a suspended transport model similar to the bed load models used in this study, with the lower step lengths used for bed load and the higher step lengths used for suspended load. With such a method, it's important to be able to determine how the transported sediment is divided among the bed load and suspended modes.

\section{CONCLUSION \& FUTURE WORK}

In this study we were able to find a step length model that allows our dune evolution model to find simi- 
lar behaviour as the more complex model of Shimizu et al. (2009). For that, we adjusted the step length model. Most importantly we allow step lengths to become higher, though we are still well below the values predicted by the equation found by Sekine \& Kikkawa (1992). This results in that our model can predict a transition to upper-stage plane bed and clearly shows hysteresis effects. While the associated moments in time are predicted close to the results of Shimizu et al. (2009) the exact results in terms of water depth are somewhat different.

In the future we will test the model with scenarios from the flume, and we will see how well it works for field conditions. Possibly the model will be extended with a suspended load model as well.

\section{ACKNOWLEDGMENT}

This study is carried out as part of the project 'BedFormFlood', supported by the Technology Foundation STW, the applied science division of NWO and the technology programme of the Ministry of Economic Affairs.

The authors are grateful to dr. Marjolein Dohmen-Janssen and dr. Jan Ribberink for their support and discussions.

\section{REFERENCES}

Allen, J.R.L. (1978). Computational methods for dune timelag: Calculations using Stein's rule for dune height. Sedimentary Geology 20(3): 165-216.

Casas, A., G. Benito, V.R. Thorndycraft, M. Rico (2006). The topographic data source of digital terrain models as a key element in the accuracy of hydraulic flood modelling. Earth Surface Processes and Land Forms 31: 444-456.

Einstein, H.A. (1950). The bed load function for sediment transportation in open channel flows. Technical bulletin, No. 1026, U.S. Department of Agriculture, Soil Conservation Service.

Engelund, F. (1970). Instability of erodible beds. Journal of Fluid Mechanics 42: 225-244.

Fernandez Luque, R. and R. Van Beek (1976). Erosion and transport of bed sediment. Journal of hydraulic research: 14 (2): 127-144.

Francis, J.R.D. (1973). Experiment on the motion of solitary grains along the bed of a water stream. Proceedings of the Royal Society of London A332: 443-471.

Fredsøe, J. 1974. On the development of dunes in erodible channels. Journal of Fluid Mechanics 64: 1-16.

Hulscher, S.J.M.H. (1996). Tidal-induced large-scale regular bedform patterns in a three-dimensional shallow water model. Journal of Geophysical Research 101: 20,72720,744 .

Julien, P.Y. and G.J. Klaassen, 1995. Sand-dune geometry of large rivers during floods. Journal of Hydraulic Engineering 121(9): 657-663.

Kennedy, J.F. (1963). The mechanics of dunes and antidunes in erodible-bed channels. Journal of Fluid Mechanics 16: 521-544.
Meyer-Peter, E. and R. Müller (1948). Formulas for bed-load transport. Proceedings of the 2nd IAHR congress 2: 39-64.

Morvan, H., D. Knight, N. Wright, X. Tang, A. Crossley (2008). The concept of roughness in fluvial hydraulics and its formulation in $1 \mathrm{D}, 2 \mathrm{D}$ and $3 \mathrm{D}$ numerical simulation models. Journal of Hydraulic Research 46(2): 191-208.

Nabi, M., H.J. De Vriend, E. Mosselman, C.J. Sloff, Y. Shimi$\mathrm{zu}$ (2010) - Simulation of subaqueous dunes using detailed hydrodynamics. RCEM 2009.

Nabi, M., H.J. de Vriend, E. Mosselman, C.J. Sloff, and Y. Shimizu (2013), Detailed simulation of morphodynamics: 3. Ripples and dunes. Water Resource Research 49: 1-14.

Nakagawa, H. \& T. Tsujimoto (1980). Sand bed instability due to bed load motion. Journal of the Hydraulics Division 106(12): 2029-2051.

Nelson, J.M., A.R. Burman, Y. Shimizu, S.R. McLean, R.L. Shreve, M. Schmeeckle (2005). Computing flow and sediment transport over bedforms. RCEM 2005 (2): 861-872.

Németh, A.A., S.J.M.H. Hulscher, R.M.J. Van Damme (2006). Simulating offshore sand waves. Coastal Engineering 53: 265-275.

Paarlberg, A.J., C.M. Dohmen-Janssen, S.J.M.H. Hulscher, P. Termes (2007). A parameterization of flow separation over subaqueous dunes. Water Resource Research 43, W12417.

Paarlberg, A.J., C.M. Dohmen-Janssen, S.J.M.H. Hulscher, and A.P.P. Termes (2009). Modelling river dune evolution using a parameterization of flow separation. Journal of $\mathrm{Ge}$ ophysical Research. Pt. F: Earth surface 114, F01014.

Paarlberg, A.J., C.M. Dohmen-Janssen, S.J.M.H. Hulscher, P. Termes, R. Schielen (2010). Modelling the effect of timedependent river dune evolution on bed roughness and stage. Earth Surfaces Processes and Landforms 35: 1854-1866.

Sekine, M., and H. Kikkawa (1984). Transportation mechanism of bed-load in an open channel. Proceedings of the Japanese Society of Civil Engineering 351: 69-75 (in Japanese).

Sekine, M., and H. Kikkawa (1992). Mechanics of Saltating Grains II. Journal of Hydraulic Engineering 118(4): 536558.

Shimizu, Y., S. Giri, I. Yamaguchi, J. Nelson (2009). Numerical simulation of dune-flat bed transition and stagedischarge relationship with hysteresis effect. Water Resources Research, 45.

Tjerry, S. \& J. Fredsøe (2005). Calculation of dune morphology. Journal of Geophysical Research - Earth Surface 110.

Van den Berg, J. (2012). Non-linear process based modeling of offshore sand waves. Continental Shelf Research 37: 26-35.

Van Duin, O.J.M., J.S. Ribberink, C.M. Dohmen-Janssen and S.J.M.H. Hulscher (2014, submitted). Which bed load transport formulations allow modelling the transition from dunes to upper-stage plane beds?

Van Duin, O.J.M., J.S. Ribberink, C.M. Dohmen-Janssen and S.J.M.H. Hulscher (2012). Particle step length variation along river dunes. River Flow 2012.

Van Rijn, L.C. (1984). Sediment transport part III: Bedforms and alluvial roughness. Journal of Hydraulic Engineering 110(12): 1733-1754.

Vidal, J.-P., S. Moisan, J.-B. Faure, D. Dartus (2007). River model calibration, from guidelines to operational support tools. Environmental Modelling \& Software 22: 16281640 .

Yalin, M.S. (1964). Geometrical properties of sand waves. Journal of the Hydraulic Division 90(5).

Yamaguchi, S. \& N. Izumi (2002). Weakly nonlinear stability analysis of dune formation. Proceedings of River Flow 2002: 843-850. 